\title{
Overexpression of the anti-apoptotic protein BAG3 in human choroidal melanoma: A case report
}

\author{
TATSUYA YUNOKI $^{1}$, YOSHIAKI TABUCHI ${ }^{2}$, TAKASHI KONDO $^{3}$, YOKO ISHII $^{4}$ and ATSUSHI HAYASHI ${ }^{1}$ \\ ${ }^{1}$ Department of Ophthalmology, Graduate School of Medicine and Pharmaceutical Sciences, University of Toyama; \\ ${ }^{2}$ Division of Molecular Genetics Research, Life Science Research Center, University of Toyama; \\ Departments of ${ }^{3}$ Radiological Sciences and ${ }^{4}$ Pathology, Graduate School of Medicine and \\ Pharmaceutical Sciences, University of Toyama, Toyama 930-0194, Japan
}

Received October 5, 2016; Accepted March 14, 2017

DOI: $10.3892 / \mathrm{ol} .2017 .5958$

\begin{abstract}
Bcl-2-associated athanogene 3 (BAG3), a co-chaperone of heat shock protein 70 (HSP70), exerts anti-apoptotic effects in various malignant tumors. However, relationships between choroidal melanoma and BAG3 are poorly studied. This study investigated the expression of BAG3 in a case of human choroidal melanoma. Funduscopy, computed tomography, and single-photon emission computed tomography with the intravenous injection of $\mathrm{N}$-isopropyl- $p-\left[{ }^{123} \mathrm{I}\right]$ iodoamphetamine strongly indicated choroidal melanoma in a 68 -year-old woman. Accordingly, we carried out an enucleation and pathological diagnosis. Proteins and total RNA were extracted from normal retinochoroidal and tumor tissues. Proteins were also extracted from ocular nevus tissues of other patients. We examined the expression of BAG3 protein and mRNA using Western blotting and the real-time quantitative polymerase chain reaction, respectively. Immunohistochemical stains were positive for melan-A, HMB-45, and S-100. Histopathology confirmed a choroidal melanoma. The expression of BAG3 protein and mRNA in the choroidal melanoma tissue was upregulated with respect to both normal retinochoroidal tissue and ocular nevus tissues from other patients. Because BAG3 may inhibit apoptosis of choroidal melanoma and facilitate its
\end{abstract}

Correspondence to: Dr Tatsuya Yunoki, Department of Ophthalmology, Graduate School of Medicine and Pharmaceutical Sciences, University of Toyama, 2630 Sugitani, Toyama 930-0194, Japan

E-mail: yunokiki@med.u-toyama.ac.jp

Abbreviations: BAG3, Bcl-2-associated athanogene 3; GAPDH, glyceraldehyde 3-phosphate dehydrogenase; HSF1, heat shock factor 1; HSP70, heat shock protein 70; HSPs, heat shock proteins; qPCR, quantitative polymerase chain reaction; SD, standard deviation

Key words: choroidal melanoma, Bcl-2 associated athanogene 3, heat shock proteins, melan-A, single-photon emission computed tomography, ocular nevus survival, overexpression of this gene product may be a prognostic marker and therapeutic target.

\section{Introduction}

Choroidal melanoma is the most common intraocular tumor in adults, and it metastasizes mainly to the liver (1). Poor prognosis is related to various clinical factors such as tumor size (2). Furthermore, various molecular factors are associated with poor prognosis $(3,4)$.

Heat shock proteins (HSPs) function as molecular chaperones and exert cytoprotective effects. Among the HSPs, proteins from the HSP70 family play central roles as molecular chaperones. Bcl-2-associated athanogene 3 (BAG3) belongs to a family of co-chaperones that interacts with the ATPase domain of HSP70 (5). Although BAG3 is expressed weakly in normal cells, it is overexpressed in various malignant tumors (6-14). In melanoma cells, BAG3 is upregulated, and exerts cell survival and anti-apoptotic effects (15-17). However, relationships between choroidal melanoma and BAG3 are poorly studied. Therefore, we investigated the expression of BAG3 in human choroidal melanoma as compared to normal and ocular nevus tumor tissues.

\section{Case report}

Patients and clinical materials. A 68-year-old woman was referred to Toyama University Hospital for further evaluation of a left intraocular mass. Funduscopy revealed a pigmented choroidal mass in the temporal fundus of her left eye. B-Mode ultrasonography revealed a choroidal protrusion (Fig. 1A). Computed tomography revealed an enhanced intraocular mass (Fig. 1B). Single-photon emission computed tomography revealed a high accumulation of $\mathrm{N}$-isopropyl- $p-\left[{ }^{123} \mathrm{I}\right]$ iodoamphetamine after its intravenous injection (Fig. 1C) (18).

To definitively treat this strongly suspected case of choroidal melanoma, we enucleated the eye. Immunohistochemical stains were positive for melan-A (Fig. 2), HMB-45, and S-100 (not shown) (19). Histopathology confirmed choroidal melanoma without vascular or optic nerve invasion. Additionally, we surgically resected a conjunctival tumor from a 44-year-old man (Fig. 3A) and a lid tumor from a 74-year-old man 

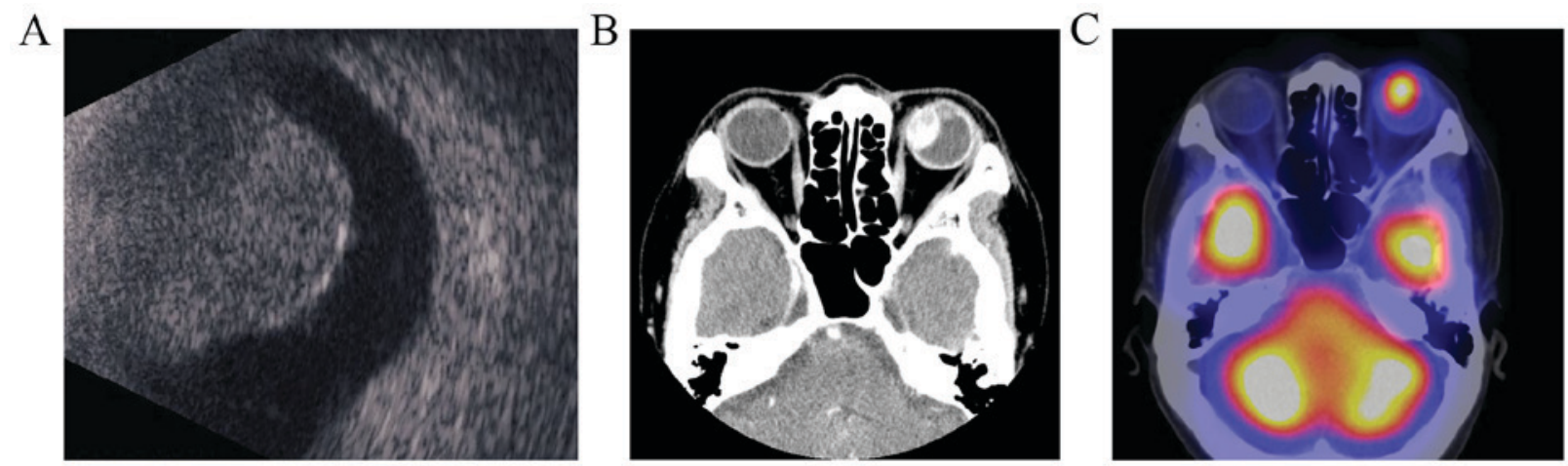

Figure 1. Images used to diagnose choroidal melanoma. (A) B-Mode ultrasonography reveals a choroidal protrusion. (B) Computed tomography reveals an enhanced intraocular mass. (C) Single-photon emission computed tomography reveals a high accumulation of N-isopropyl-p-[ $\left.{ }^{[23} \mathrm{I}\right]$ iodoamphetamine after intravenous injection.

Table I. Nucleotide sequences of primers for target genes.

\begin{tabular}{llc}
\hline Genes & Nucleotide sequence (5'-3') & GenBank accession no. \\
\hline Bcl-2-associated athanogene 3 & & \\
$\begin{array}{l}\text { Sense } \\
\text { Antisense }\end{array}$ & CGACCAGGCTACATTCCCAT & NM_004281 \\
Glyceraldehyde 3-phosphate dehydrogenase & TCTGGCTGAGTGGTTTCTGG & \\
$\begin{array}{l}\text { Sense } \\
\text { Antisense }\end{array}$ & AAGGCTGGGGCTCATTTGCA & NM_002046 \\
\hline
\end{tabular}

(Fig. 3B); these tumors were diagnosed as conjunctival nevus and lid nevus, respectively.

Normal retinochoroidal and melanoma tissue samples were obtained from the enucleated eye (Fig. 4), and nevus tissue samples were obtained from the resected tumor tissues (Fig. 3A and B). Our procedures conformed to the tenets of the World Medical Association's Declaration of Helsinki. Written informed consent was obtained from the patients after provision of sufficient information about the procedures.

Western blotting. Protein extracts were prepared by homogenizing tissue samples in a lysis buffer $(150 \mathrm{mM} \mathrm{NaCl}$, $1 \%$ Nonidet P-40, and $50 \mathrm{mM}$ Tris-HCl, $\mathrm{pH}$ 8.0) containing a protease inhibitor cocktail (Nacalai Tesque, Kyoto, Japan). After electrophoresis on sodium dodecyl sulfate-polyacrylamide gels, proteins were transferred electrophoretically onto polyvinylidene fluoride membranes. The following primary antibodies were used: rabbit monoclonal anti-BAG3 (GTX62327; GeneTex Inc., Irvine, CA, USA); mouse monoclonal anti-HSP70 (SR-B810; MBL, Nagoya, Japan); rabbit monoclonal anti anti-HSF1 (GTX62022; GeneTex Inc.) and mouse monoclonal anti-glyceraldehyde 3-phosphate dehydrogenase (GAPDH) (MAB374; Millipore, Temecula, CA, USA). The immunoreactive proteins were visualized using a luminescence image analyzer (LAS 4000mini; GE Healthcare, Tokyo, Japan) with an enhanced chemiluminescence detection system. GAPDH served as the loading control.

RNA isolation. Using an RNeasy Total RNA Extraction kit (Qiagen K.K., Tokyo, Japan), total RNA was extracted from tissue samples and treated with on-column DNase I (RNase-free DNase kit, Qiagen K.K.) (20).

Quantitative polymerase chain reaction $(q P C R)$. $\mathrm{qPCR}$ was performed on a Real-Time PCR Mx3005P system (Agilent Technologies, Santa Clara, CA, USA) using a SYBR PreMix ExTaq kit (Takara Bio, Inc., Shiga, Japan). The relevant primer sequences are listed in Table I. mRNA expression levels for each protein were normalized to the mRNA expression level for GAPDH (20).

Statistical analysis. Measurements are reported as means \pm standard deviations. Student's t-test was used for statistical analysis, and $\mathrm{P}<0.05$ was considered statistically significant.

\section{Results}

To analyze the involvement of BAG3 within a choroidal melanoma, we examined its protein and mRNA expression levels using western blotting and qPCR, respectively. The BAG3 protein level in the human choroidal melanoma tissue was upregulated compared to that in normal retinochoroidal tissue (Fig. 5A). Furthermore, as observed using Western blotting, the expression levels of heat shock factor 1 (HSF1) and HSP70 were upregulated in human choroidal melanoma relative to expression levels in normal retinochoroidal tissues (Fig. 5A). Similarly, qPCR indicated that the BAG3 mRNA level in the human choroidal melanoma tissue was significantly higher than that in normal retinochoroidal tissue $(\mathrm{n}=4, \mathrm{P}=0.000291)$ 

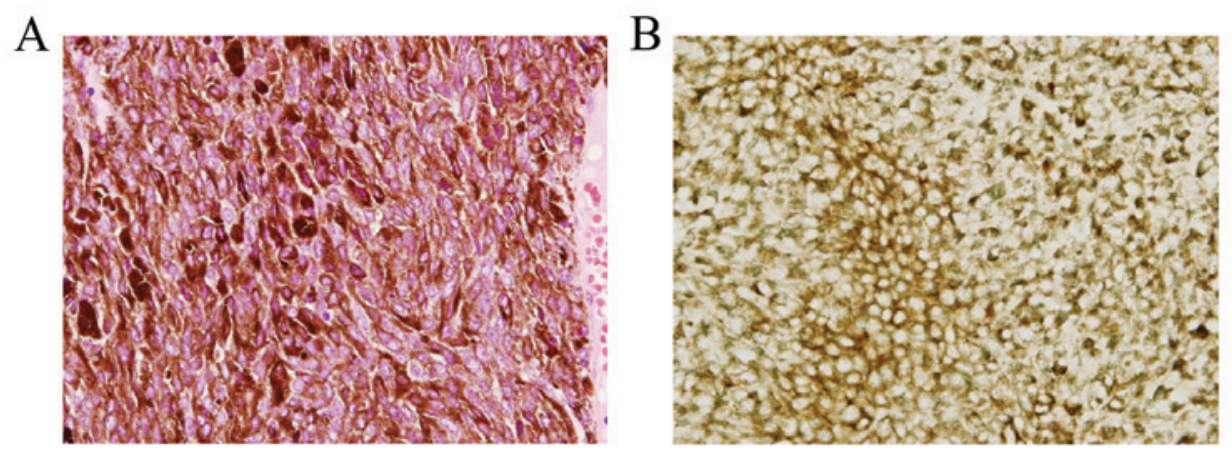

Figure 2. Histopathologic analysis of choroidal melanoma. (A) Hematoxylin and eosin stain of choroidal melanoma (original magnification, x40). (B) The melanoma stain is strongly positive for melan-A (original magnification, $\mathrm{x} 40$ ).
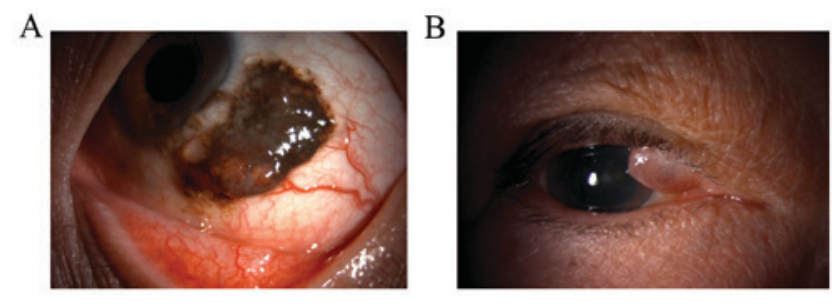

Figure 3. Photographs of (A) a conjunctival nevus of a 44-year-old man and (B) a lid nevus of a 74-year-old man.

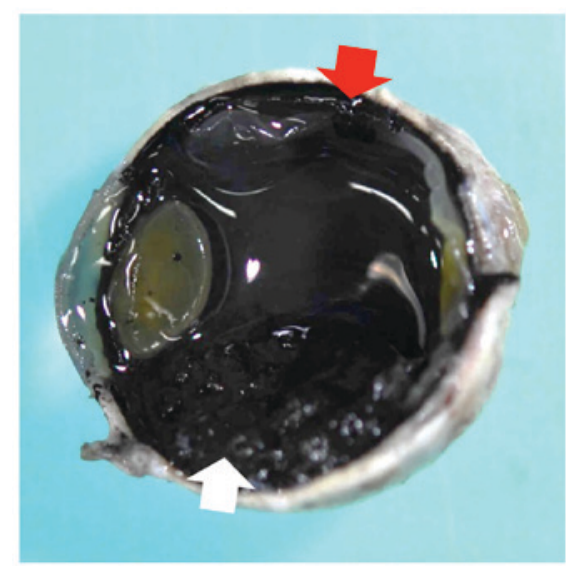

Figure 4. The enucleated eye includes normal retinochoroidal tissue (red arrow) and melanoma tissue (white arrow).

(Fig. 5B). Additionally, we confirmed BAG3 expression in choroidal melanoma using immunohistochemical analysis (Fig. 5C).

Western blots also indicated that the BAG3 level in the human choroidal melanoma tissue was upregulated compared to those in nevus tissue samples from other patients. Moreover, BAG3 levels in the conjunctival nevus were higher than those in the lid nevus (Fig. 6). These findings suggest that BAG3 was upregulated in the human choroidal melanoma relative to normal retinochoroidal and nevus tissues.

\section{Discussion}

The mechanisms of choroidal melanoma progression and metastasis remain poorly understood, and treatment options are limited. Regardless of the progress of diagnostic technology,

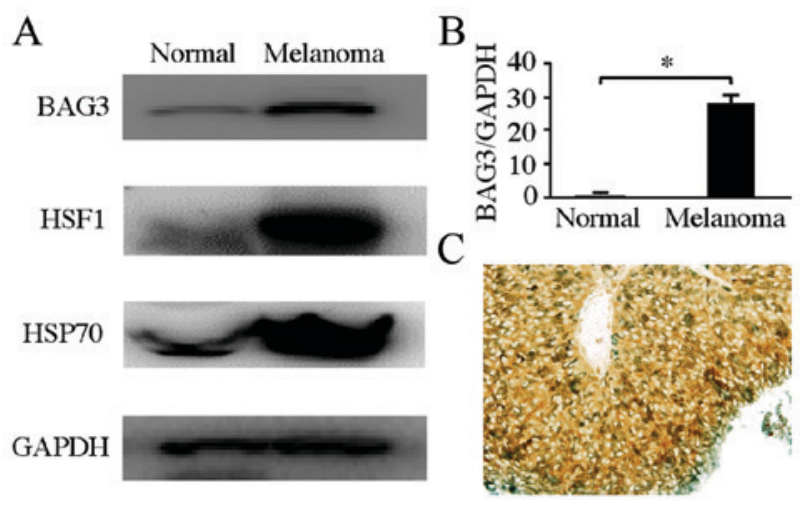

Figure 5. Expression levels of BAG3 in choroidal melanoma and normal retinochoroidal tissues. (A) BAG3, HSF1 and HSP70 proteins were determined by Western blotting. (B) BAG3 mRNA was determined by the quantitative polymerase chain reaction (qPCR). Western blotting used primary antibodies specific for BAG3, HSF1, HSP70 and GAPDH. GAPDH served as the loading control. The qPCR assay was performed with specific primers for BAG3 and GAPDH. The BAG3 mRNA level was normalized to the GAPDH expression level. Measurements are reported as means \pm standard deviations $(\mathrm{n}=4)$. ${ }^{*} \mathrm{P}<0.05$. (C) The melanoma stain is strongly positive for BAG3 in histopathological analysis (original magnification, $\mathrm{x} 40$ ).

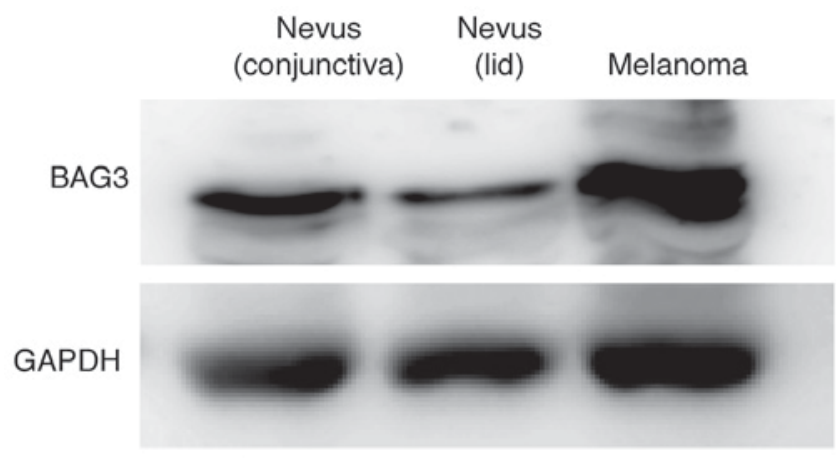

Figure 6. Expression levels of BAG3 protein in choroidal melanoma and nevus tissues. Western blotting was performed using primary antibodies specific for BAG3 and GAPDH. GAPDH served as the loading control.

choroidal melanoma causes death due to liver metastasis (21). Accordingly, the study of choroidal melanoma-specific biomarkers is important for improving prognosis accuracy.

It is thought that an association between the heat shock response and melanoma is important. HSF1 is required for 
melanoma invasion and metastasis (22). BAG3, a co-chaperone of HSP70, is overexpressed in multiple malignant tumors and exerts anti-apoptotic effects (15-17). Observations in vitro and in vivo indicate that the induction of BAG3 is at least partly mediated by the activation of HSF1 (23). In this study, BAG3 levels were upregulated via HSF1 activation in human choroidal melanoma relative to its expression levels in normal retinochoroidal tissues. However, little is known about the anti-apoptotic role of BAG3 in human choroidal melanoma. To our knowledge, we are the first to report overexpression of BAG3 protein and mRNA in human choroidal melanoma relative to expression levels in normal retinochoroidal and ocular nevus tissues. Franco et al reported that BAG3 levels in eye melanoma are relatively low, but are related to metastasis at other sites (15). It is possible that BAG3-positive choroidal melanoma is associated with a poor prognosis. We think that careful follow-up of patients is necessary in BAG3-positive choroidal melanoma.

Similar to its effects in other malignant tumors, BAG3 may contribute to survival through anti-apoptotic activity in choroidal melanoma. We believe that BAG3 may be a prognostic marker and therapeutic target. Further investigation is necessary to understand the relationships between choroidal melanoma and BAG3.

In conclusion, BAG3 is overexpressed in human choroidal melanoma relative to other related tissues. Our findings suggest that BAG3 may offer a therapeutic target for patients with choroidal melanoma.

\section{Acknowledgements}

This study was supported in part by a Grant-in-Aid for Scientific Research (16K20309) from the Japan Society for the Promotion of Science.

\section{References}

1. Wöll E, Bedikian A and Legha SS: Uveal melanoma: Natural history and treatment options for metastatic disease. Melanoma Res 9: 575-581, 1999.

2. Shields CL, Furuta M, Thangappan A, Nagori S, Mashayekhi A, Lally DR, Kelly CC, Rudich DS, Nagori AV, Wakade OA, et al: Metastasis of uveal melanoma millimeter-by-millimeter in 8033 consecutive eyes. Arch Ophthalmol 127: 989-998, 1999.

3. Mooy CM and De Jong PT: Prognostic parameters in uveal melanoma: A review. Surv Ophthalmol 41: 215-228, 1996.

4. Scholes AG, Damato BE, Nunn J, Hiscott P, Grierson I and Field JK: Monosomy 3 in uveal melanoma: Correlation with clinical and histologic predictors of survival. Invest Ophthalmol Vis Sci 44: 1008-1011, 2003.

5. Takayama S, Xie Z and Reed JC: An evolutionarily conserved family of Hsp70/Hsc70 molecular chaperone regulators. J Biol Chem 274: 781-786, 1999

6. Liao Q, Ozawa F, Friess H,Zimmermann A, Takayama S, Reed JC, Kleeff $\mathrm{J}$ and Büchler MW: The anti-apoptotic protein BAG-3 is overexpressed in pancreatic cancer and induced by heat stress in pancreatic cancer cell lines. FEBS Lett 503: 151-157, 2001.

7. Kassis JN, Virador VM, Guancial EA, Kimm D, Ho AS, Mishra M, Chuang EY, Cook J, Gius D and Kohn EC: Genomic and phenotypic analysis reveals a key role for CCN1 (CYR61) in BAG3-modulated adhesion and invasion. J Pathol 218: 495-504, 2009.
8. Chiappetta G, Ammirante M, Basile A, Rosati A, Festa M, Monaco M, Vuttariello E, Pasquinelli R, Arra C, Zerilli M, et al: The antiapoptotic protein BAG3 is expressed in thyroid carcinomas and modulates apoptosis mediated by tumor necrosis factor-related apoptosis-inducing ligand. J Clin Endocrinol Metab 92: 1159-1163, 2007.

9. Wang HQ, Liu BQ, Gao YY, Meng X, Guan Y, Zhang HY and Du ZX: Inhibition of the JNK signalling pathway enhances proteasome inhibitor-induced apoptosis of kidney cancer cells by suppression of BAG3 expression. Br J Pharmacol 158: 1405-1412, 2009.

10. Suzuki M, Iwasaki M, Sugio A, Hishiya A, Tanaka R, Endo T, Takayama S and Saito T: BAG3 (BCL2-associated athanogene 3) interacts with MMP-2 to positively regulate invasion by ovarian carcinoma cells. Cancer Lett 303: 65-71, 2011.

11. Tang JT, Wang JL, Du W, Hong J, Zhao SL, Wang YC, Xiong H, Chen HM and Fang JY: MicroRNA 345, a methylation-sensitive microRNA is involved in cell proliferation and invasion in human colorectal cancer. Carcinogenesis 32: 1207-1215, 2011.

12. Festa M, Del Valle L, Khalili K, Franco R, Scognamiglio G, Graziano V, De Laurenzi V, Turco MC and Rosati A: BAG3 protein is overexpressed in human glioblastoma and is a potential target for therapy. Am J Pathol 178: 2504-2512, 2011.

13. Liu P, Xu B, Li J and Lu H: BAG3 gene silencing sensitizes leukemic cells to Bortezomib-induced apoptosis. FEBS Lett 583: 401-406, 2009.

14. Romano MF, Festa M, Pagliuca G, Lerose R, Bisogni R, Chiurazzi F, Storti G, Volpe S, Venuta S, Turco MC and Leone A: BAG3 protein controls B-chronic lymphocytic leukaemia cell apoptosis. Cell Death Differ 10: 383-385, 2003.

15. Franco R, Scognamiglio G, Salerno V, Sebastiani A, Cennamo G, Ascierto PA, Botti G, Turco MC and Rosati A: Expression of the anti-apoptotic protein BAG3 in human melanomas. J Invest Dermatol 132: 252-254, 2012

16. Basile A, Zeppa R, Pasquino N, Arra C, Ammirante M, Festa M, Barbieri A, Giudice A, Pascale M, Turco MC and Rosati A: Exposure to $50 \mathrm{~Hz}$ electromagnetic field raises the levels of the anti-apoptotic protein BAG3 in melanoma cells. J Cell Physiol 226: 2901-2907, 2011.

17. Ammirante M, Rosati A, Arra C, Basile A, Falco A, Festa M, Pascale M, d'Avenia M, Marzullo L, Belisario MA, et al: IKK $\{$ gamma\} protein is a target of BAG3 regulatory activity in human tumor growth. Proc Natl Acad Sci USA 107: 7497-7502, 2010.

18. Goto H: Clinical efficacy of 123I-IMP SPECT for the diagnosis of malignant uveal melanoma. Int J Clin Oncol 9: 74-78, 2004.

19. Levasseur SD, Paton KE, Van Raamsdonk CD, Heran MK and White VA: Mutation of GNAQ in a cytologically unusual choroidal melanoma in an 18-month-old child. JAMA Ophthalmol 131: 810-812, 2013.

20. Tabuchi Y, Yunoki T, Hoshi N, Suzuki N and Kondo T: Genes and gene networks involved in sodium fluoride-elicited cell death accompanying endoplasmic reticulum stress in oral epithelial cells. Int J Mol Sci 15: 8959-8978, 2014.

21. Spanknebel K, Coit DG, Bieligk SC, Gonen M, Rosai J and Klimstra DS: Characterization of micrometastatic disease in melanoma sentinel lymph nodes by enhanced pathology: Recommendations for standardizing pathologic analysis. Am J Surg Pathol 29: 305-317, 2005.

22. Nakamura Y, Fujimoto M, Fukushima S, Nakamura A, Hayashida N, Takii R, Takaki E, Nakai A and Muto M: Heat shock factor 1 is required for migration and invasion of human melanoma in vitro and in vivo. Cancer Lett 354: 329-335, 2014.

23. Franceschelli S, Rosati A, Lerose R, De Nicola S, Turco MC and Pascale M: Bag3 gene expression is regulated by heat shock factor 1. J Cell Physiol 215: 575-577, 2008. 